\title{
A single mode porous-core square lattice photonic crystal fiber for $\mathrm{THz}$ wave propagation
}

\author{
Md. Rabiul Hasan 1*, Md. Ariful Islam² and Ahmmed A. Rifat ${ }^{3}$
}

\begin{abstract}
Background: Interests on low-loss terahertz ( $\mathrm{THz}$ ) waveguides are increasing due to their remarkable applications in various fields. Since most the materials are highly absorbent to $\mathrm{THz}$ waves therefore it is an ongoing challenge to obtain a low-loss waveguide. This paper presents a novel porous-core square lattice photonic crystal fiber (PCF) for efficient transmission of $\mathrm{THz}$ waves.

Methods: The guiding properties of the proposed fiber are characterized by using finite element method (FEM) with circular perfectly matched layer (PML) boundary conditions.

Results: It is demonstrated that the designed PCF shows very low effective material loss (EML) of $0.076 \mathrm{~cm}^{-1}$ at 1.0 $\mathrm{THz}$ that indicates about $62 \%$ reduction of bulk absorption loss of the background material. In addition to this, the proposed fiber exhibits low confinement loss of $8.96 \times 10^{-3} \mathrm{~dB} / \mathrm{cm}$ and low flattened dispersion of $0.96 \pm 0.086$ $\mathrm{ps} / \mathrm{THz} / \mathrm{cm}$ for the optimal design parameters. Other important propagation characteristics such as single mode propagation, power fraction, and bending loss are also investigated thoroughly.
\end{abstract}

Conclusions: A porous-core PCF is an efficient mechanism for the transmission of THz waves. The proposed low-loss and low-dispersion PCF can find numerous applications in THz regime.

Keywords: Terahertz guidance, Rffective material loss, Confinement loss, Single mode propagation, Finite element method

\section{Background}

In recent years, enormous efforts have been paid to $\mathrm{THz}$ PCFs due to their applications in multidisciplinary fields including remote sensing [1], imaging [2], security screening [3] and $\mathrm{THz}$ time domain spectroscopy [4]. Development of low-loss and low dispersion THz PCFs enable variety of promising applications in biomedical technology such as non-invasive early diagnosis of skin cancer including the basal cell carcinoma [5], dysplastic skin nevi and melanomas of hardly-accessible skin areas [6]. Moreover, it can be employed for minimally-invasive diagnosis of colon tissue cancers [7] and intraoperative diagnosis of breast tumors [8]. Most of the THz communication systems use free space medium to propagate

\footnotetext{
* Correspondence: rabiul.hasan@ruet.ac.bd

'Department of Electronics \& Telecommunication Engineering, Rajshahi

University of Engineering \& Technology, Rajshahi, Bangladesh

Full list of author information is available at the end of the article
}

$\mathrm{THz}$ waves, which suffer from various types of problems. Strong water vapor absorption, misalignment issues and complex integration with other components are the major obstacles for efficient transportation of $\mathrm{THz}$ waves [9]. Researchers around the world develop various forms of waveguide structures to overcome these problems. However, due to the challenge of dealing with excessive absorption loss, the commercial production of efficient $\mathrm{THz}$ waveguide is lagging behind. A great deal of numerical and experimental researches has been performed in order to increase the reliability during the transmission of broadband $\mathrm{THz}$ waves through a guiding structure. For example, the theoretical characteristics and experimental measurements of groove guide structure have been demonstrated for low-loss $\mathrm{THz}$ signal transmission [10]. Mitrofanov et al. experimentally proposed a dielectric-lined hollow metallic waveguide for low transmission loss and low group velocity 
dispersion [11]. The attenuation and dispersion properties of coplanar transmission line (CTL) in the THz frequency bands have been experimentally presented in [12]. Wang et al. proposed bare metal wire waveguide having low average attenuation coefficient of $0.03 \mathrm{~cm}^{-1}$ [13]. Besides, PCF having background material as Teflon exhibited high absorption loss of $0.3 \mathrm{~cm}^{-1}$ at $1 \mathrm{THz}$ [14]. The plastic photonic crystal fiber (PPCF) by Han et al. showed comparatively higher loss about $0.5 \mathrm{~cm}^{-1}$ at 0.6 $\mathrm{THz}$ [15]. Recently, silicon photonic-crystal slab waveguide has been demonstrated having extremely low propagation loss of $0.04 \mathrm{~dB} / \mathrm{cm}$ at $0.33 \mathrm{THz}$ [16]. Very recently, PCF based on sapphire shaped crystal has been proposed for efficient THz guidance. This PCF showed low power extinction coefficient of $0.02 \pm 0.015 \mathrm{~dB} / \mathrm{cm}$ at $1.45 \mathrm{THz}$ with minimal dispersion between 1.0 and $1.55 \mathrm{THz}$ [17].

Recently, tremendous success has been achieved in engineering of the guiding properties of $\mathrm{THz}$ fibers by using relatively smaller air holes (smaller than operating wavelength) in the core of the PCF. Such types of structures are known as porous-core PCFs. Porous-core PCFs have gained increased attention due to freedoms for tuning EML, dispersion and confinement loss in a smart way [18]. The wave propagation through porous-core PCF is based on the non-absorbing nature of dry air. Using this formulation, making a tight light confinement in the core and sending most of the mode power into the porous-air holes can greatly reduce the absorption and confinement loss. With this in mind, a few remarkable PCFs have been reported to date. A porous-core honeycomb $\mathrm{THz}$ fiber has been proposed by Bao et al.
[19] that exhibited low material absorption loss of $1.5 \mathrm{~dB} / \mathrm{cm}$ at $1.0 \mathrm{THz}$. However, maintaining of periodicity during the fabrication of honeycomb structure is too difficult. Porous-core octagonal PCF has been reported by Kaijage et al. [20] that showed low EML of $0.07 \mathrm{~cm}^{-1}$ at $1.0 \mathrm{THz}$. In this work, the authors have not considered few crucial guiding characteristics such as dispersion and power fraction. A hexagonal PCF with hexagonal porous-air holes has been proposed in [21], where the reported EML was comparatively higher about $0.12 \mathrm{~cm}^{-1}$. Very recently, a porous-core kagome lattice PCF has been reported in [22] using hexagonal lattice air-holes in the core. This PCF showed very low EML of $0.035 \mathrm{~cm}^{-1}$ at $1.0 \mathrm{THz}$. However, owing to complex structure fabrication of kagome lattice PCF is limited. The structures proposed in Refs. [23-25] also represent noticeable improvement in terms of EML, confinement loss and power fraction. Although promising porous core PCFs have been proposed based on the triangular lattice, a square lattice PCF has never been reported in the literature. Therefore, it is quite interesting to observe how the guiding properties of square lattice differ from a regular triangular lattice. The square lattice PCF permits easy fabrication process since it can be realized with standard stack and draw technique [26, 27]. Moreover, technological feasibility of the square lattice PCF has already been demonstrated in Ref. [28].

In this paper, to the best of our knowledge for the first time, we introduce a new design that consists of square lattice in both outer cladding and inner porous-core. It has been demonstrated that by properly tailoring the fiber's design parameters EML can be lowered significantly, while

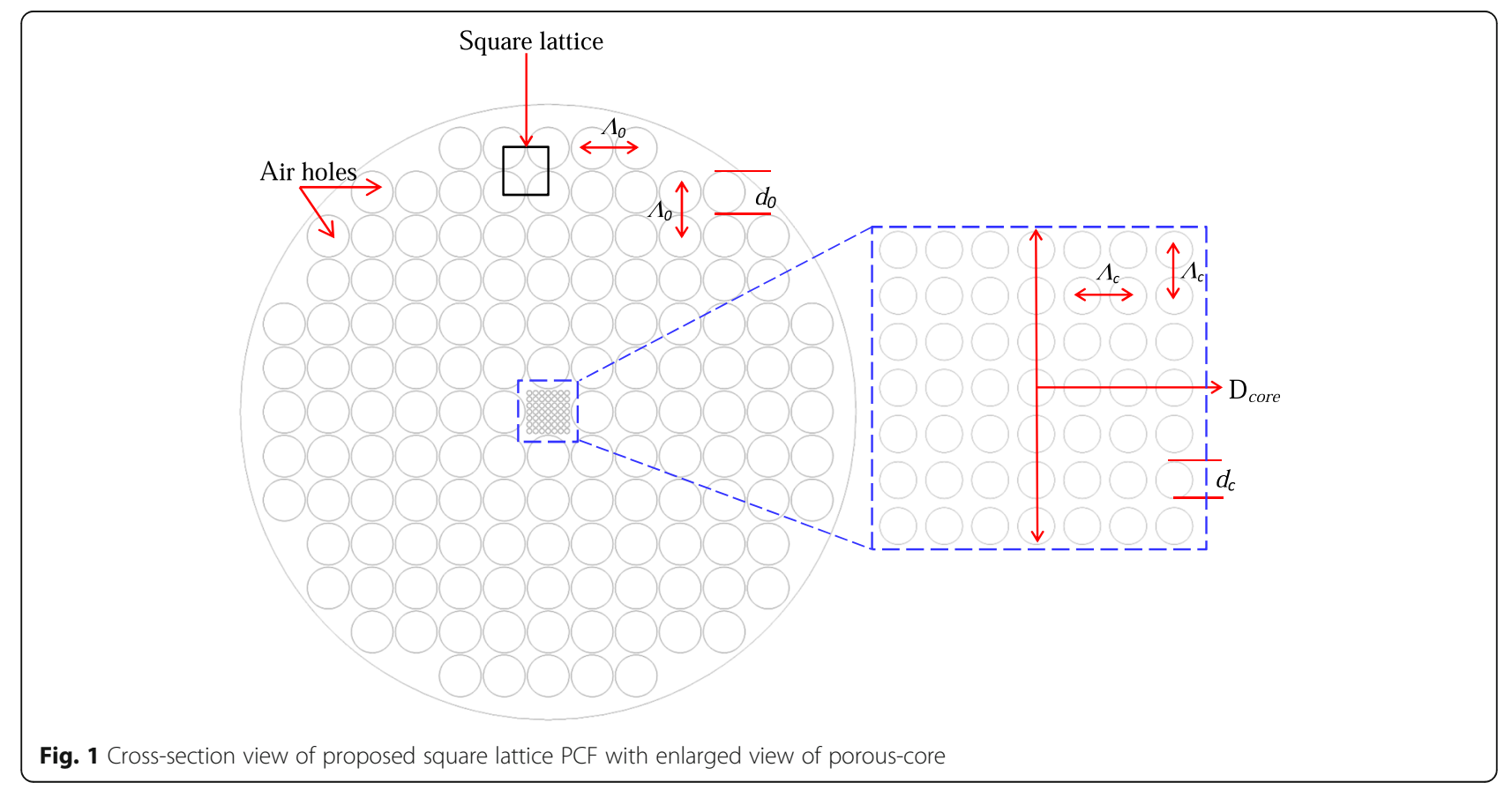


other guiding parameters such as confinement loss and power fraction can be kept above acceptable limits. In addition to these, single mode guidance and bending loss characteristics of the proposed structure has been analyzed rigorously. The design methodology of the proposed PCF has been discussed in section 2, simulation results and discussions are explained in section 3 , and finally conclusion is summarized in section 4 .

\section{Design guidelines}

The design schematic of the proposed square lattice PCF is shown in Fig. 1 with enlarged view of porous-core. As clearly shown in the figure, square lattice has been used in both outer cladding and inner porous core to form the complete structure. Here, both outer and inner layer is formed by circular air holes with isosceles square unit lattices. It is seen from Fig. 1 that, $\mathrm{D}_{\text {core }}$ indicates the side length of inner square lattice. The hole-to-hole distance and diameter of air holes in the outer lattice are denoted by $\Lambda_{0}$ (pitch) and $d_{0}$, respectively. On the other hand, hole-tohole distance and diameter of air holes in the inner lattice are denoted by $\Lambda_{\mathrm{c}}$ and $d_{\mathrm{c}}$, respectively. It is important to note that the value of pitch in the same ring and two adjacent rings are kept identical which is also indicated in Fig. 1. In this structure, a relationship is made between $\Lambda_{0}$ and $\Lambda_{\mathrm{c}}$ to $\mathrm{D}_{\text {core }}$ so that changing of $\mathrm{D}_{\text {core }}$ can automatically adjust the value of inner and outer pitch. These parameters are related by $\Lambda_{0}=\mathrm{D}_{\text {core }} / 1.5$ and $\Lambda_{\mathrm{c}}=\mathrm{D}_{\text {core }} / 10.5$, respectively. For a preset value of $D_{\text {core }}$, several simulations were done to adjust the pitch values. The pitch values have carefully selected so that air holes of core and cladding region do not overlap each other. Moreover, selection of these pitch values provides best results in terms of EML and power fraction. It should be mentioned that using other values of $\Lambda_{0}$ and $\Lambda_{\mathrm{c}}$ might result poor confinement of light in the porous-core and higher value of EML. The air filling fraction (AFF) in the outer lattice $d / \Lambda_{0}$ is kept constant as 0.95. This higher AFF results lower refractive index around the core, which makes strong light confinement in the porous-core region [23]. The diameter of inner air-holes $d_{\mathrm{c}}$ is determined by the porosity, which can be defined as the ratio between total areas of porous air holes to area of core. The number of air-hole rings $\left(N_{r}\right)$ is chosen as 6 since this value is sufficient to provide low confinement loss.

The whole PCF is based on cyclic-olefin copolymer (COC), which is commercially known as TOPAS. Compared to other polymer materials (such as PMMA, HDPE, PP etc.), TOPAS provides few definite advantages. It shows nearly constant refractive index over $0.1-2 \mathrm{THz}$ frequencies [19]. Moreover, it shows low dispersion, low confinement loss and good protection against water vapor absorption [29].

\section{Methods}

The commercially available FEM based on state-of-the-art COMSOL v.5.0 has been used to design and characterize of the proposed porous-core square lattice PCF. To calculate the confinement loss a circular perfectly matched layer (PML) boundary condition has been imposed in the outer cladding. About 58,546 triangular elements and 6,124 edge elements have been used to represent the complete mesh. The average element quality has used about 0.96 . The minimum element size has been fixed about $0.81 \mu \mathrm{m}$, which is enough for mapping different sizes of air holes. Throughout the simulation, finer element size has been used to obtain greater degree of accuracy. During the simulation the bulk material absorption loss of $0.20 \mathrm{~cm}^{-1}$ has been inserted, which is comparable with the experimental results reported in [19]. Dry air is the most transparent medium for $\mathrm{THz}$ waves having almost no absorption $\left(\alpha_{\mathrm{m}}=0\right)$ in $\mathrm{THz}$ frequency bands $[18,30]$. Therefore, absorption loss of air has not been taken into account during the calculation of different losses. It should be mentioned that losses of the proposed fiber have been studied for $\mathrm{THz}$ electromagnetic wave intensity.

\section{Results and discussions}

The fundamental mode field profile of the proposed PCF for $50 \%, 65 \%$ and $80 \%$ core porosities are shown

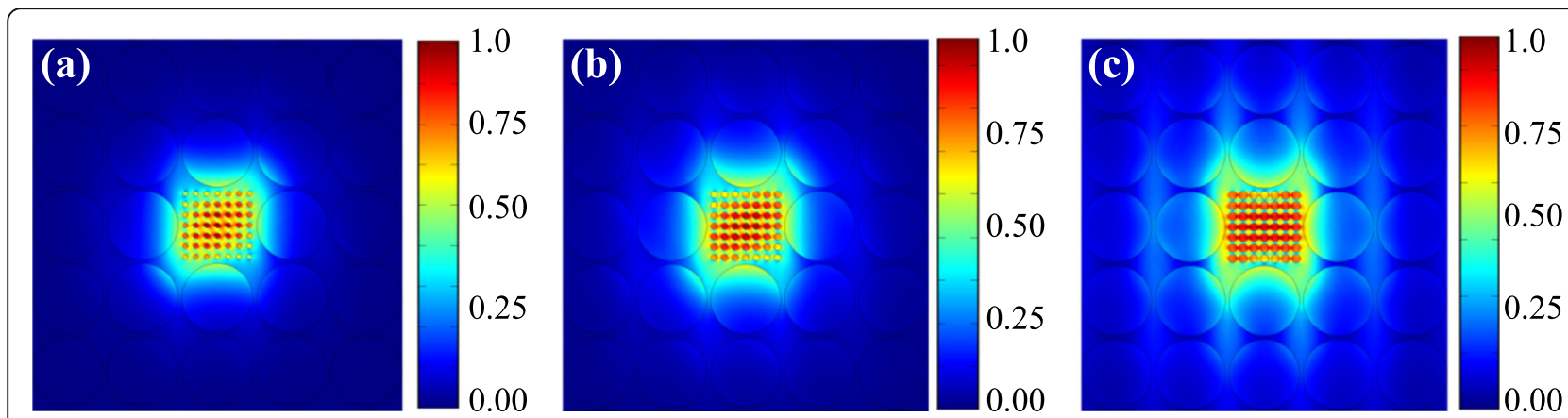

Fig. 2 Mode field distribution of the proposed square lattice PCF for (a) $50 \%$ porosity (b) $65 \%$ porosity and (c) $80 \%$ porosity at $D_{\text {core }}=280 \mu m$ and $f=1 \mathrm{THz}$. (The scale of color bar indicates the normalized values) 
in Fig. 2. It can be seen from the figure that mode fields are bounded in the porous-core region, which is essential for efficient $\mathrm{THz}$ wave propagation. One can observe from Fig. 2 that increasing the core porosity results spreading of mode power towards the cladding region. Since increasing of core porosity causes reduction of index contrast between core and cladding, the guiding modes tend to spread into the cladding region.

Most of the polymer materials are highly absorbent in $\mathrm{THz}$ frequency bands and effective transmission of $\mathrm{THz}$ signal is mostly limited by the so called EML. The EML is often quantified by the following expression [30]

$$
\alpha_{\text {eff }}=\frac{\left(\varepsilon_{0} / \mu_{0}\right)^{1 / 2} \int_{A_{\text {mat }}} n \alpha_{\text {mat }}|E|^{2} d A}{2 \int_{\text {All }} S_{z} d A} \mathrm{~cm}^{-1},
$$

where $\varepsilon_{O}$ and $\mu_{O}$ indicate the permittivity and permeability of vacuum, $n$ indicates the refractive index of TOPAS, $\alpha_{\text {mat }}$ indicates the bulk material absorption loss, and $S_{z}$ denotes the $z$ component of the Poynting vector. Here, $S_{z}=1 / 2 \operatorname{Re}\left(\mathrm{E} \times \mathrm{H}^{*}\right) z$, where $\mathrm{E}$ is the electric field component and $\mathrm{H}^{\prime \prime}$ is the complex conjugate of magnetic field component. The integration of the numerator of Eq. (1) has performed over solid material region of TOPAS and denominator has performed over all regions. EML as a function of core diameter $\left(\mathrm{D}_{\text {core }}\right)$ for three different core porosities is illustrated in Fig. 3. It can be seen from the figure that at a fixed $D_{\text {core }}$ the value of EML can be reduced by using higher core porosities. On the other hand, at a fixed core porosity the value of EML increases when $\mathrm{D}_{\text {core }}$ is made larger. The physical explanation behind this phenomenon can be explained by the mode field profiles in Fig. 2. When core porosity is scaled up, the amount of solid material portion decreases correspondingly. As a consequent, the value of

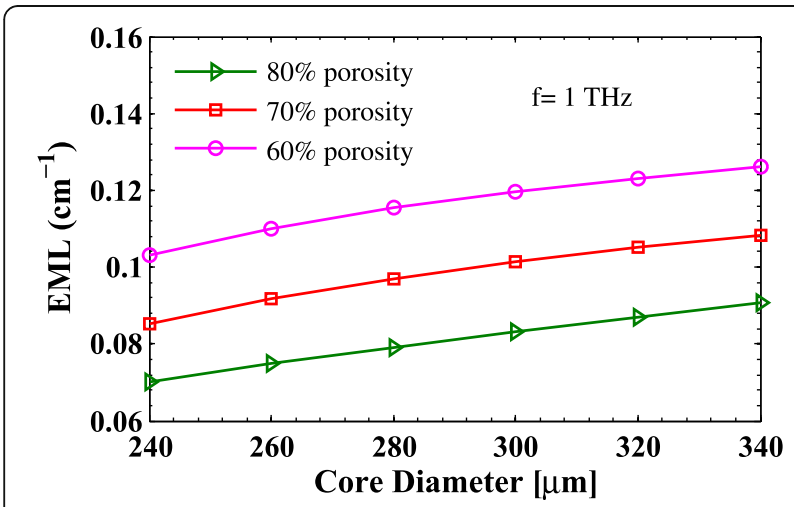

Fig. 3 EML of the proposed square lattice PCF as a function of core diameters for different core porosities at $f=1 \mathrm{THz}$
EML is reduced. Again, enlarging the value of $\mathrm{D}_{\text {core }}$ results increasing of solid material area in the core therefore EML increases. At $\mathrm{D}_{\text {core }}=280 \mu \mathrm{m}$, porosity $=80 \%$ and $f=1 \mathrm{THz}$ the EML has found about $0.076 \mathrm{~cm}^{-1}$. This value is comparable with previously published results in Refs [20, 21, 23, 25].

EML as a function of operating frequency for different core porosities is shown in Fig. 4. As seen from the figure that at fixed frequency, EML can be reduced by using higher core porosities. When core porosity is increased, the guiding electromagnetic wave interacts with less amount of material. As a result, EML of the proposed fiber decreases. The lowest EML has found about $0.064 \mathrm{~cm}^{-1}$ when $f=0.8 \mathrm{THz}, \mathrm{D}_{\text {core }}=280 \mu \mathrm{m}$ and porosity $=80 \%$.

Due to finite number of air holes used in the cladding region, light confinement in PCF is inherently leaky. Confinement loss of a THz PCF is an important guiding property since it potentially limits the length of real $\mathrm{THz}$ transmission system. Confinement loss of a PCF can be calculated using the following equation [25]

$$
\alpha_{C L}=8.686 \times \frac{2 \pi f}{c} \operatorname{Im}\left(n_{e f f}\right) \mathrm{dB} / \mathrm{cm},
$$

where $f$ is the operating frequency, $c$ is the speed of light and $\operatorname{Im}\left(n_{\text {eff }}\right)$ is the imaginary part of the effective refractive index. Confinement loss as a function of operating frequency for different core porosities is depicted in Fig. 5. It can be evident that confinement loss decreases rapidly with increasing of frequency. One can also observe that same results can be obtained by scaled down of core porosities. This is obvious since at higher frequencies mode power tightly constricts at the porous core region. On the other hand, when core porosity is scaled down the guiding mode sees less air volume. This way index contrast increases, which is responsible for significant reduction of confinement loss. With $\mathrm{D}_{\text {core }}=280 \mu \mathrm{m}$,

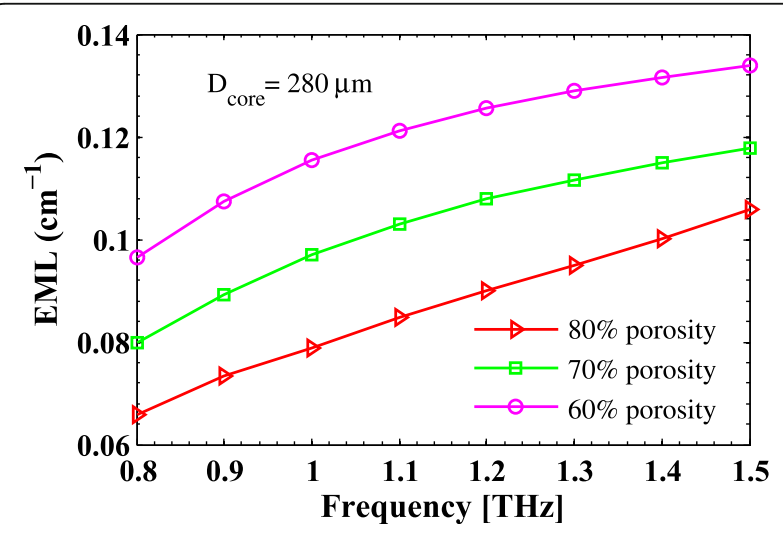

Fig. 4 EML of the proposed square lattice PCF as a function of varying frequencies for different core porosities at $D_{\text {core }}=280 \mu \mathrm{m}$ 


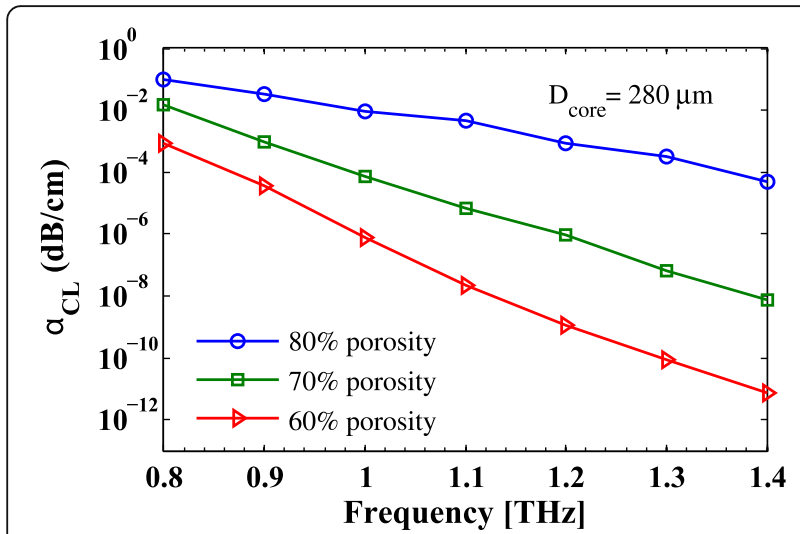

Fig. 5 Confinement loss of the proposed square lattice PCF as a function of varying frequencies for different core porosities at $D_{\text {core }}=280 \mu \mathrm{m}$

$\mathrm{f}=1 \mathrm{THz}$ and porosity $=80 \%$ the calculated confinement loss is about $8.96 \times 10^{-3} \mathrm{~dB} / \mathrm{cm}$.

Power fraction represents the amount of total mode power propagating in various regions of a PCF. This parameter can be calculated using the following formula [25]

$$
\text { Power fraction }=\frac{\int_{X} S_{Z} d A}{\int_{A l l} S_{Z} d A},
$$

where $X$ in the numerator denotes the area of region of interest and denominator denotes the total area. For example, power fraction in air core refers to the amount of total mode power propagates in the air holes of the porous core. Figure 6 shows the confinement loss and power fraction in air holes of the porous core as a function $\mathrm{D}_{\text {core }}$ with different core porosities. Since majority of mode power confines in the porous core, we consider

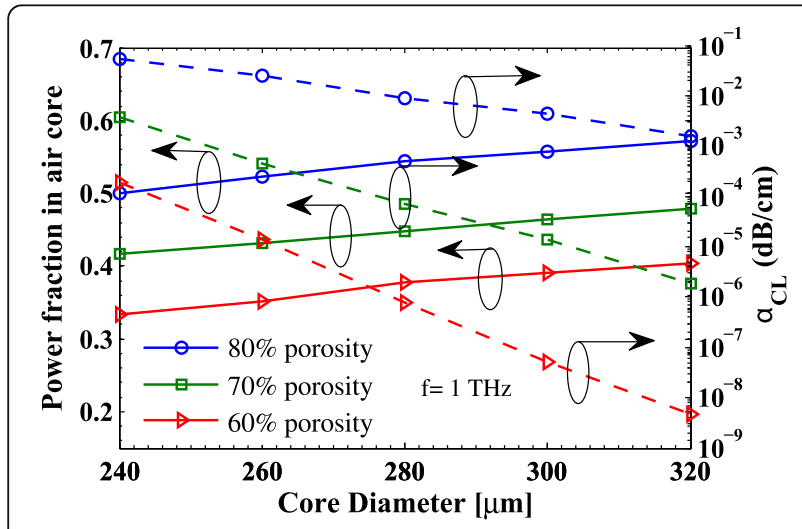

Fig. 6 Power fraction in air-core and confinement loss of the proposed square lattice PCF as a function of core diameters for different core porosities at $f=1 \mathrm{THz}$ only the power fraction in air holes of the porous core. It can be evident from Fig. 6 that at a fixed value of $\mathrm{D}_{\text {core }}$, power fraction in core air holes can be increased by scaling up of the core porosity. Increasing the value of core porosity leads to increase the amount of air volume in the porous-core region. For this reason, mode power mostly propagates through porous air holes and increases the power fraction of air holes in the core. It has been found that about $53 \%$ of total power confines in the air core when $\mathrm{D}_{\text {core }}=280 \mu \mathrm{m}$, porosity $=80 \%$ and $f=1 \mathrm{THz}$. Figure 6 also shows the confinement loss characteristics as a function $\mathrm{D}_{\text {core }}$. As illustrated in the figure that at the same $\mathrm{D}_{\text {core }}$, confinement loss increases with scaling up of core porosity. When core porosity is increased, guiding mode tends to spread towards the cladding region (illustrated in Fig. 2). As a consequent, confinement loss of the proposed fiber increases.

The single mode condition of the proposed porouscore PCF can be determined by using V parameter (normalized frequency). $\mathrm{V}$ parameter of a PCF can be expressed by the following relation [9]

$$
V=\frac{2 \pi r f}{c} \sqrt{n_{c o}^{2}-n_{c l}^{2}} \leq 2.405
$$

where $r$ is the half of the side length, $f$ is the operating frequency, $n_{c o}$ is the refractive index of core and $n_{c l}$ is the refractive index of cladding. Due to porous structure, the value of $n_{c o}$ is assumed to be the effective index $\left(\mathrm{n}_{e f f}\right)$ of the guiding mode [31]. The value of $\mathrm{n}_{\text {eff }}$ can be obtained from the simulation software directly. On the other hand, due to high concentration of air-holes in the cladding region, the value of $n_{c l}$ can be considered as the refractive index of air $\left(n_{c l}=1\right)$ [31]. V parameter as a function of frequency with $\mathrm{D}_{\text {core }}=280 \mu \mathrm{m}$, porosity $=80 \%$ is shown in Fig. 7. It can be noted that the value of $\mathrm{V}$ parameter increases with increasing of frequency. It has

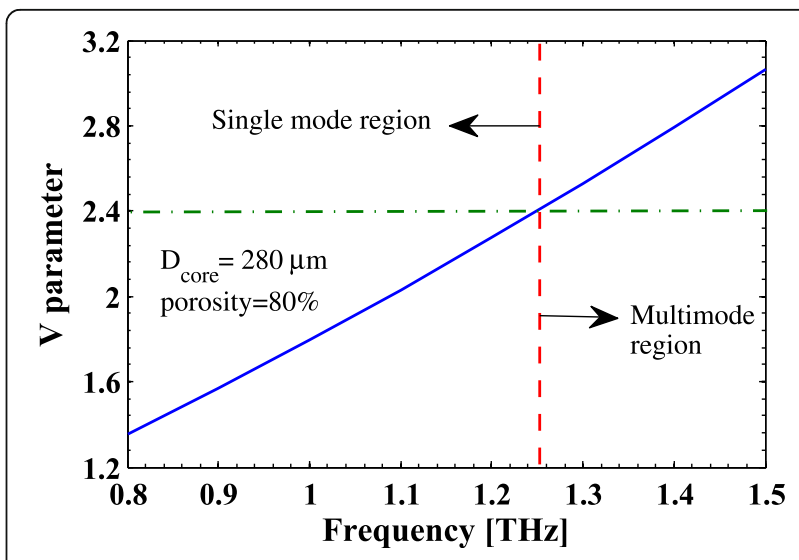

Fig. $7 \mathrm{~V}$ parameter of the proposed square lattice PCF as a function of frequency for $\mathrm{D}_{\text {core }}=280 \mu \mathrm{m}$ and porosity $=80 \%$ 
been observed that increasing of frequency causes significant increase of index contrast between effective indices of core and cladding therefore $\mathrm{V}$ parameter is increased. From the Fig. 7, one can clearly notice that mode cutoff occurs at $f=1.24 \mathrm{THz}$. Therefore, the proposed fiber acts as a single mode fiber up to $1.24 \mathrm{THz}$ with $\mathrm{D}_{\text {core }}=280 \mu \mathrm{m}$ and porosity $=80 \%$. V parameter as function of core porosity with $\mathrm{D}_{\text {core }}=280 \mu \mathrm{m}$ and $f=1 \mathrm{THz}$ is shown in Fig. 8. It is seen from the figure that $\mathrm{V}$ parameter decreases with scaling up of core porosities. The reason is the reduction of effective index difference between core and cladding. It has been found that mode cutoff occurs when core porosity is below $36.3 \%$. The single mode and multimode regions are clearly indicated by the arrow symbols. Now, $\mathrm{V}$ parameters as a function of $\mathrm{D}_{\text {core }}$ with $f=1 \mathrm{THz}$ and porosity $=80 \%$ is shown in Fig. 9. It can be observed that $\mathrm{V}$ parameter increases with increasing of $\mathrm{D}_{\text {core }}$. Here, mode cutoff occurs at $\mathrm{D}_{\text {core }}=348.4 \mu \mathrm{m}$ when $f=1 \mathrm{THz}$ and porosity $=80 \%$. The above discussion reflects that the proposed porouscore PCF acts as a single mode fiber when $\mathrm{D}_{\text {core }}=280 \mu \mathrm{m}$, porosity $=80 \%$ and $f=1 \mathrm{THz}$.

Dispersion plays a vital role when it comes to consider in practical implementations. A PCF should show very small dispersion over a wide range of frequencies, which is particularly suitable for transmission of wideband signals [22]. On the contrary, flattened dispersion profile permits transmission of simultaneous signals having approximately same pulse spreading. As already discussed that TOPAS exhibits nearly constant refractive index between 0.1 and $1.5 \mathrm{THz}$. Due to constant refractive index the induced material dispersion can be assumed to zero. Therefore, we need to consider only the contribution of waveguide dispersion $\left(\beta_{2}\right)$, which is given by $[25,30,31]$

$$
\beta_{2}=\frac{2}{c} \frac{d n_{e f f}}{d \omega}+\frac{\omega}{c} \frac{d^{2} n_{e f f}}{d \omega^{2}} \mathrm{ps} / \mathrm{THz} / \mathrm{cm},
$$

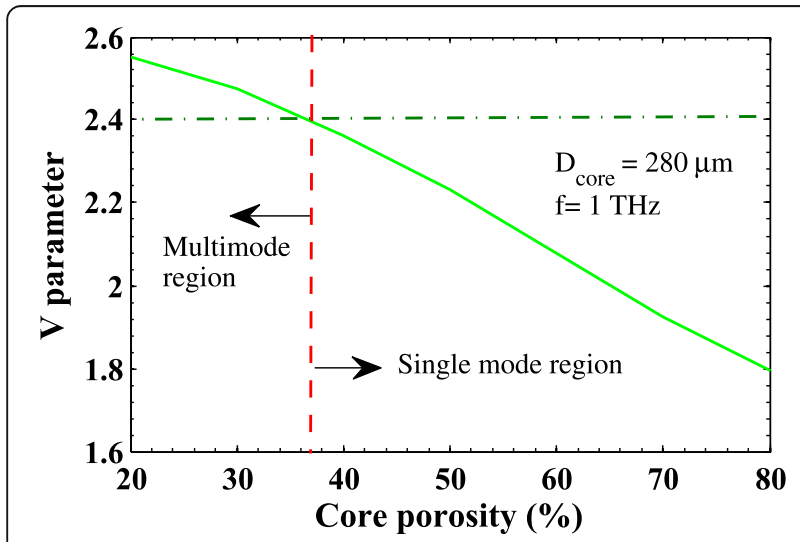

Fig. $8 \mathrm{~V}$ parameter of the proposed square lattice PCF as a function of core porosity for $\mathrm{D}_{\text {core }}=280 \mu \mathrm{m}$ and $f=1 \mathrm{THz}$

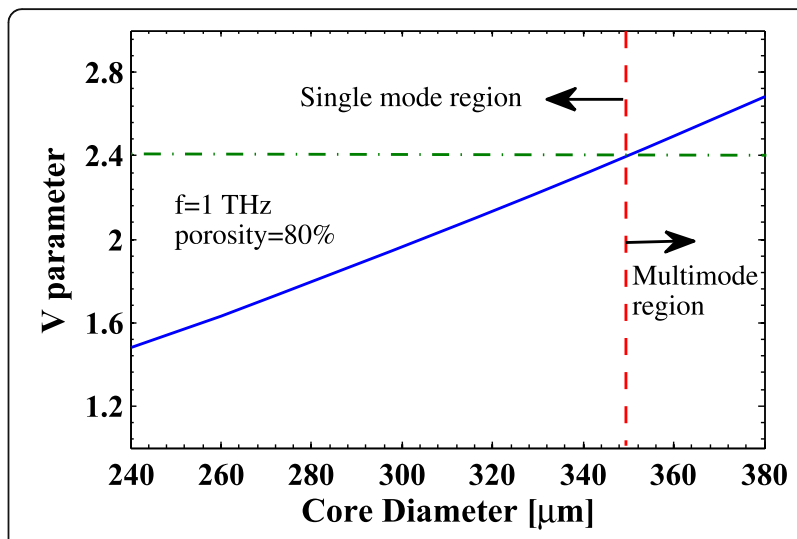

Fig. $9 \mathrm{~V}$ parameter of the proposed square lattice PCF as a function of core diameter for porosity $=80 \%$ and $f=1 \mathrm{THz}$

where $\omega$ denotes angular center frequency $\omega=2 \pi f, f$ denotes operating frequency, $n_{\text {eff }}$ denotes effective refractive index, and $c$ denotes velocity of light. Dispersion as function of operating frequency for different core porosities is depicted in Fig. 10. It can be evident that the proposed PCF shows positively flat dispersion over $0.8-1.3 \mathrm{THz}$ frequencies. Note that increasing the core porosity results decreasing of dispersion magnitude as well as dispersion variation. The lowest dispersion magnitude and dispersion variation have been found when $\mathrm{D}_{\text {core }}=280 \mu \mathrm{m}$ and porosity $=80 \%$. With these values, the dispersion magnitude at $1.0 \mathrm{THz}$ is about $0.96 \mathrm{ps} / \mathrm{THz} / \mathrm{cm}$ and dispersion variation between 0.8 and $1.3 \mathrm{THz}$ is about $\pm 0.086 \mathrm{ps} / \mathrm{THz} / \mathrm{cm}$. To our knowledge, this is the lowest dispersion variation reported in the literature of porous-core THz PCFs.

Now, the bending loss of the proposed PCF has been investigated. When a fiber is bent, mode power tends to diffuse towards the direction of bent and this way bending loss occurs. Here, the conformal transformation method has been used to calculate the bending loss [32]. In this method, the bent fiber is replaced by its

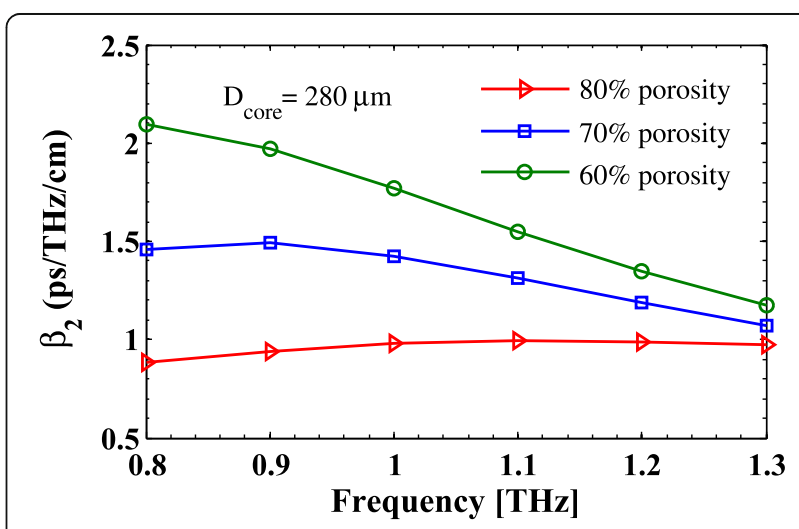

Fig. 10 Dispersion of the proposed square lattice PCF as a function of frequency for different core porosities at $D_{\text {core }}=280 \mu \mathrm{m}$ 


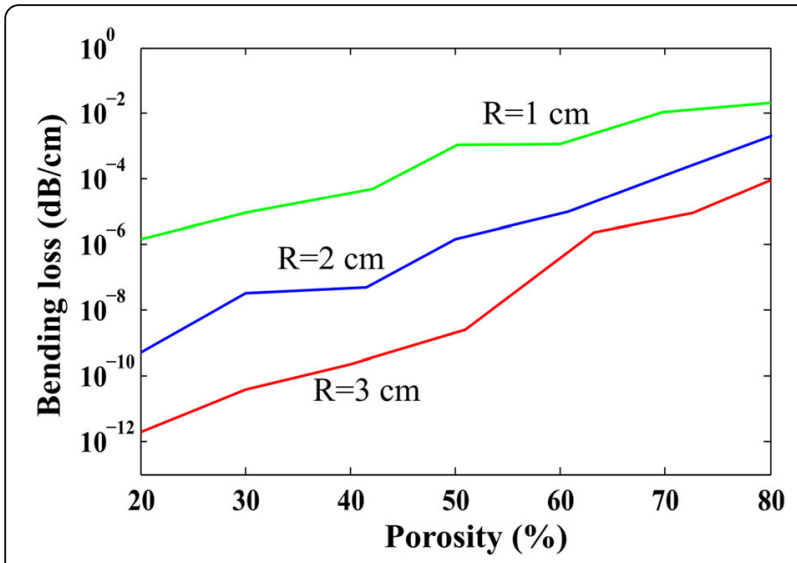

Fig. 11 Bending loss of the proposed square lattice PCF as a function of core porosity for different bend radius at $D_{\text {core }}=280 \mu \mathrm{m}$ and $f=1 \mathrm{THz}$

equivalent straight one with modified refractive index given by the following equation [31]

$$
n_{e q}(x, y)=n(x, y)\left[1+\frac{x}{R}\right]
$$

where $n_{e q}(x, y)$ is the refractive index of equivalent straight fiber, $n(x, y)$ is the original refractive index profile of the proposed fiber, $R$ is the bend radius and $x$ is the distance from center of the PCF. Bending loss as a function of core porosity with different bending radius is shown in Fig. 11. As illustrated in the figure that bending loss increases with increasing of core porosities. This is due to the fact that, at higher core porosities, guiding mode loosely bounds by the porous air core therefore it fails to trap the light much enough.

Total loss of the proposed fiber consists of effective material loss, confinement loss and bending loss. Total loss of the designed structure as a function of core porosity is shown in Fig. 12. At $\mathrm{D}_{\text {core }}=280 \mu \mathrm{m}$, porosity $=80 \%$,

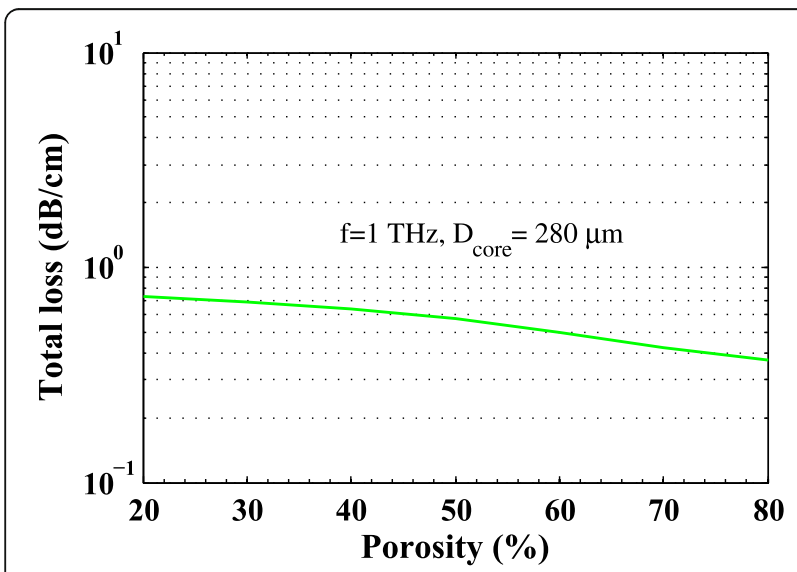

Fig. 12 Total loss of the proposed square lattice PCF as a function of core porosity for $\mathrm{D}_{\text {core }}=280 \mu \mathrm{m}$ and $f=1 \mathrm{THz}$
Table 1 Comparison among proposed porous-core PCF with other existing works

\begin{tabular}{|c|c|c|c|c|c|}
\hline Ref. & $\begin{array}{l}\mathrm{EML} \\
\left(\mathrm{cm}^{-1}\right)\end{array}$ & $\begin{array}{l}{ }^{a} \mathrm{CL} \\
(\mathrm{dB} / \mathrm{cm})\end{array}$ & $\begin{array}{l}\text { Power } \\
\text { fraction (\%) }\end{array}$ & $\begin{array}{l}\text { Total loss } \\
(\mathrm{dB} / \mathrm{cm})\end{array}$ & $\begin{array}{l}\text { Lattice type } \\
\text { (cladding-core) }\end{array}$ \\
\hline [20] & 0.07 & 0.177 & - & 0.481 & octa-octa \\
\hline [21] & 0.12 & 0.29 & 58 & 1.43 & hexa-hexa \\
\hline [31] & 0.066 & $2.05 \times 10^{-3}$ & 40 & 0.71 & $\begin{array}{l}\text { hexa-rotated } \\
\text { hexa }\end{array}$ \\
\hline This work & 0.076 & $8.96 \times 10^{-3}$ & 53 & 0.392 & square-square \\
\hline
\end{tabular}

$\mathrm{R}=1 \mathrm{~cm}$ and $\mathrm{f}=1 \mathrm{THz}$ the total loss has been found about $0.3715 \mathrm{~dB} / \mathrm{cm}$.

At this point, we would like to make a comparison table that compares guiding properties of the proposed PCF to other existing PCFs in the literature. Table 1 shows the comparison taking into account of EML, confinement loss $\left(\alpha_{\mathrm{CL}}\right)$, power fraction of porous air holes in core and dispersion variation. It is worthy to mention that all guiding properties are mentioned at 1.0 $\mathrm{THz}$ frequency.

Finally, we would like to address the fabrication possibilities of the proposed square lattice $\mathrm{THz}$ fiber. It should be noted that by using the conventional stack and draw technique it could be possible to fabricate square lattice PCF. Since this standard method has been already employed to square lattice PCFs [26-28]; therefore, this technique would be a good choice to fabricate the proposed structure. The edgedefined film-fed growth (EFG) or Stepanov technology would be another viable solution to fabricate the square lattice PCF [33]. This technology has been already employed in [17] in order to obtain high quality fiber cross section. To our knowledge, these two verified techniques [17, 33] should be enough for practical realization of the proposed square lattice PCF.

\section{Conclusion}

In summary, a new square lattice based porous-core PCF has been proposed and its guiding properties are characterized with varying of different structural parameters. A thorough numerical investigation has been performed to obtain very low EML of $0.076 \mathrm{~cm}^{-}$ 1 and low confinement loss about $8.96 \times 10^{-3} \mathrm{~dB} / \mathrm{cm}$ at $1.0 \mathrm{THz}$. The single mode propagation of the proposed design has been justified for different core porosities and core diameters. The structure is feasible in terms of fabrication due to its realistic size and contains only circular air holes. All these characteristics make this porous-core square lattice PCF a potential candidate for applications in efficient $\mathrm{THz}$ wave propagation. 


\section{Acknowledgements}

The authors would like to thank anonymous reviewers for their valuable comments to make the paper suitable for publication. The authors are grateful to Mr. Mohammad Harun Or Rashid for his help to make grammatical corrections.

\section{Funding}

The article processing charge has been waived by BioMed Central.

\section{Authors' contributions}

$\mathrm{MRH}$ designed and performed simulations, analyzed data and finally drafted the manuscript. MAI performed simulations and prepared the revised manuscript. AAR edited and finalized the manuscript to be published. All authors read and approved the final manuscript.

\section{Competing interests}

The authors declare that they have no competing interest.

\section{Author details}

'Department of Electronics \& Telecommunication Engineering, Rajshahi University of Engineering \& Technology, Rajshahi, Bangladesh. ${ }^{2}$ Department of Physics, Rajshahi University of Engineering \& Technology, Rajshahi, Bangladesh. ${ }^{3}$ Integrated Lightwave Research Group, Department of Electrical Engineering, University of Malaya, Kuala Lumpur, Malaysia.

Received: 2 May 2016 Accepted: 20 September 2016

Published online: 01 October 2016

\section{References}

1. Liu, J., Dai, J., Chin, S.L., Zhang, X.C.: Broadband terahertz wave remote sensing using coherent manipulation of fluorescence from asymmetrically ionized gases". Nat. Photonics. 4, 627-631 (2010)

2. Fukunaga, K., Sekine, N., Hosako, I., Oda, N., Yoneyama, H., Sudou, T.: Realtime terahertz imaging for art conservation science". J. Eur. Opt. Soc. Rapid Publ. 3, 08027 (2008)

3. Kawase, K., Ogawa, Y., Watanabe, Y., Inoue, H.: Non-destructive terahertz imaging of illicit drugs using spectral fingerprints". Opt. Express. 11, 2549-2554 (2003)

4. Mizuno, M., Fukunaga, K., Saito, S., Hosako, I.: Analysis of calcium carbonate for differentiating between pigments using terahertz spectroscopy". J. Eur. Opt. Soc. Rapid Publ. 4, 09044 (2009)

5. Wallace, V.P., Fitzgerald, A.J., Shankar, S., Flanagan, N., Pye, R., Cluff, J., Arnone, D.D.: Terahertz pulsed imaging of basal cell carcinoma ex vivo and in vivo". Br. J. Dermatol. 151, 424-432 (2004)

6. Zaytsev, K.I., Kudrin, K.G., Karasik, V.E., Reshetov, I.V., Yurchenko, S.O.: In vivo terahertz spectroscopy of pigmentary skin nevi: Pilot study of non-invasive early diagnosis of dysplasia". Appl. Phys. Lett. 106, 053702 (2015)

7. Reid, C.B., Fitzgerald, A., Reese, G., Goldin, R., Tekkis, P., O'Kelly, P.S.E., MacPherson, P., Gibson, A.P., Wallace, V.P.: "Terahertz pulsed imaging of freshly excised human colonic tissues". Phys. Med. Biol. 56, 4333 (2011)

8. Ashworth, P.C., MacPherson, E.P., Provenzano, E., Pinder, S.E., Purushotham, A.D., Pepper, M., Wallace, V.P.: Terahertz pulsed spectroscopy of freshly excised human breast cancer". Opt. Express. 17, 12444-12454 (2009)

9. Chen, N.N., Liang, J., Ren, L.Y.: High-birefringence, low-loss porous fiber for single-mode terahertz-wave guidance". Appl. Opt. 52, 5297-5302 (2013)

10. Harris, D.., Lee, K.W., Batt, RJ.: Low-loss single-mode waveguide for submillimetre and millimetre wavelengths". Infrared. Phys. 18, 741-747 (1978)

11. Mitrofanov, O., Tan, T., Mark, P.R., Bowden, B., Harrington, J.A.: Waveguide mode imaging and dispersion analysis with terahertz near-field microscopy". Appl. Phys. Lett. 94, 171104 (2009)

12. Frankel, M.Y., Gupta, S., Valdmanis, J.A., Mourou, G.A.: Terahertz attenuation and dispersion characteristics of coplanar transmission lines". IEEE Trans. Microw. Theory Techn. 39, 910-916 (1991)

13. Wang, K., Mittleman, D.M.: Metal wires for terahertz wave guiding". Nature. 432, 376-379 (2004)

14. Goto, M., Quema, A., Takahashi, H., Ono, S., Sarukura, N.: Teflon photonic crystal fiber as terahertz waveguide. Jpn. J. Appl. Phys. 43, L317-L319 (2004)

15. Han, H., Park, H., Cho, M., Kim, J.: Terahertz pulse propagation in a plastic photonic crystal fiber". Appl. Phys. Lett. 80, 2634-2636 (2002)

16. Tsuruda, K., Fujita, M., Nagatsuma, T.: Extremely low-loss terahertz waveguide based on silicon photonic-crystal slab". Opt. Express. 23, 31977-31990 (2015)
17. Zaytsev, K.I., Katyba, G.M., Kurlov, V.N., Shikunova, I.A., Karasik, V.E., Yurchenko, S.O.: Terahertz Photonic Crystal Waveguides Based on Sapphire Shaped Crystals". IEEE Trans. Terahertz Sci. 6, 576-582 (2016)

18. Atakaramians, S., Afshar, S.V. Fischer, B.M. Abbott, D., Monro, T.M.: Porous fibers: a novel approach to low loss THz waveguides". Opt. Express. 16, 8845-8854 (2008)

19. Bao, H.L., Nielsen, K., Rasmussen, H.K., Jepsen, P.U., Bang, O.: Fabrication and characterization of porous-core honeycomb bandgap THz fibers". Opt. Express. 20, 29507-29517 (2012)

20. Kaijage, S.F., Ouyang, Z., Jin, X.: Porous-core photonic crystal fiber for low loss terahertz wave guiding". IEEE Photon. Technol. Lett. 25, 1454-1457 (2013)

21. Uthman, M., Rahman, B.M.A., Kejalakshmy, N., Agarwal, A., Grattan, K.T.V.: Design and characterization of low-loss photonic crystal fiber". IEEE Photon. J. 4, 2315-2325 (2012)

22. Hasan, M.R., Anower, M.S., Hasan, M.I., Razzak, S.M.A.: Polarization maintaining low-loss slotted core kagome lattice THz fiber". IEEE Photon. Technol. Lett. 28, 1751-1754 (2016)

23. Hasan, M.I., Razzak, S.M.A., Hasanuzzaman, G.K.M., Habib, M.S.: Ultra-low material loss and dispersion flattened fiber for THz transmission". IEEE Photon. Technol. Lett. 26, 2372-2375 (2014)

24. Hassani, A., Dupuis, A., Skorobogatiy, M.: Low loss porous terahertz fibers containing multiple subwavelength holes". Appl. Phys. Lett. 92, 071101 (2008)

25. Hasan, M.R., Anower, M.S., Islam, M.A., Razzak, S.M.A.: Polarizationmaintaining low-loss porous-core spiral photonic crystal fiber for terahertz wave guidance". Appl. Opt. 55, 4145-4152 (2016)

26. Poli, F., Foroni, M., Bottacini, M., Fuochi, M., Burani, N., Rosa, L., Cucinotta, A., Selleri, S.: Single-mode regime of square-lattice photonic crystal fibers". J. Opt. Soc. Am. A 22, 1655-1661 (2005)

27. Bouk, A.H., Cucinotta, A., Poli, F., Selleri, S.: Dispersion properties of square-lattice photonic crystal fibers". Opt. Express. 12, 941-946 (2004)

28. Russell, P.S.J., Marin, E., Díez, A., Guenneau, S., Movchan, A.B.: Sonic band gaps in PCF preforms: enhancing the interaction of sound and light". Opt. Express. 11, 2555-2560 (2003)

29. Nielsen, K., Rasmussen, H.K., Adam, A.J.L., Planken, C.M., Bang, P.O., Jepsen, P. U.: Bendable, low-loss topas fibers for the terahertz frequency range". Opt. Express. 17, 8592-8601 (2009)

30. Liang, J., Ren, L., Chen, N., Zhou, C.: Broadband, low-loss, dispersion flattened porous-core photonic bandgap fiber for terahertz $(\mathrm{THz})$ wave propagation". Opt. Commu. 295, 257-261 (2013)

31. Islam, R., Hasanuzzaman, G.K.M., Habib, M.S., Rana, S., Khan, M.A.G.: Low-loss rotated porous core hexagonal single-mode fiber in THz regime". Opt. Fiber Technol. 24, 38-43 (2015)

32. Heiblum, M., Harris, J.: Analysis of curved optical waveguides by conformal transformation". IEEE J. Quantum Electron. 11, 75-83 (1975)

33. Antonov, P.I., Kurlov, V.N.: New advances and developments in the Stepanov method for the growth of shaped crystals". Crystallogr. Reports. 47, S43-S52 (2002)

\section{Submit your manuscript to a SpringerOpen ${ }^{\odot}$ journal and benefit from:}

- Convenient online submission

- Rigorous peer review

- Immediate publication on acceptance

- Open access: articles freely available online

- High visibility within the field

- Retaining the copyright to your article

Submit your next manuscript at $>$ springeropen.com 